\title{
Anterior odontoid screw fixation extrusion as reason for oesophagus perforation
}

\author{
Paula Piątek ${ }^{1 \star}$, Andrzej Maciejczak ${ }^{1,2 \star}$ \\ ${ }^{1}$ Department of Neurosurgery, St Lukas Hospital, Tarnow, Poland \\ ${ }^{2}$ Medical Faculty, University of Rzeszow, Poland
}

\section{ABSTRACT}

Background. Type Il odontoid fractures are mostly encountered in the elderly. Due to a high risk of non-union fractures in the case of conservative treatment, surgical fixation is widely recommended. Anterior odontoid screw fixation (AOSF) is a method that allows for a wide range of cervical mobility, and it is a relatively safe procedure that is recommended as the method of choice, although rare complications can be fatal when it leads to life-threatening oesophageal perforation.

Purpose. The aim of this study is to present potential risk factors which lead to these rare complications, and possible methods of treatment.

Methods. This article presents the case of a patient hospitalised in the Neurosurgery Department of St Lukas Hosital in Tarnów in 2016. A literature review was performed using PubMed; search criteria included the phrases 'odontoid fracture perforation' and 'anterior cervical spine perforation'. The search returned 235 articles, of which 55 publications were in line with the subject of this paper, with only 12 deemed appropriate for consideration.

Result. The authors present the case of an elderly patient with a history of odontoid fracture. Ten weeks after primary AOSF, the patient came to the Neurosurgery Department due to expectorating screws. This implied the need for further examination and even oesophageal reconstructive surgery or another spinal surgery. In laryngological examination and in gastroscopy there were no signs of fistula. In this case conservative treatment was proceeded. Due to odontoid fracture, non-union cervical posterior stabilisation was necessary.

Conclusion. Patients with oesophageal perforation should be treated with special care.

Key words: anterior odontoid screw fixation, oesophagus perforation, odontoid fracture

(Neurol Neurochir Pol 2021; 55 (2): 227-229)

\section{Background and importance}

Elderly patients who undergo surgical treatment are at higher risk. This is connected with the lower resistance and lower regenerative potential of their tissue. It manifests in the higher risk of non-union fractures, and intra- or postoperative perforation $[1,2]$. Decisions about surgical treatment must be well thought through, especially in elderly patients. Due to a relatively low risk and high chance of union, anterior stabilisation is recommended in type II odontoid fractures [1, 3].
A rare but serious and life-threatening complication is oesophageal perforation $[1,2,4,5]$. The strategy for treatment mainly depends on the size and location of the fistula. If the fistula is relatively small, conservative treatment is adequate. In extensive perforations, oesophageal reconstruction is required. In simpler cases, suture may be sufficient. Nevertheless, in more extensive cases vasculised flap implantation is necessary. The screws and implant should be removed [5-8]. Additional neurosurgical treatment depends on the state of healing of the fracture.

Address for correspondence: Paula Piątek, Department of Neurosurgery, St Lukas Hospital, Wałowa 33/5 Str., 33-100 Tarnów, Poland,

e-mail: paulapiatek89@gmail.com

*Both authors contributed equally to this work 


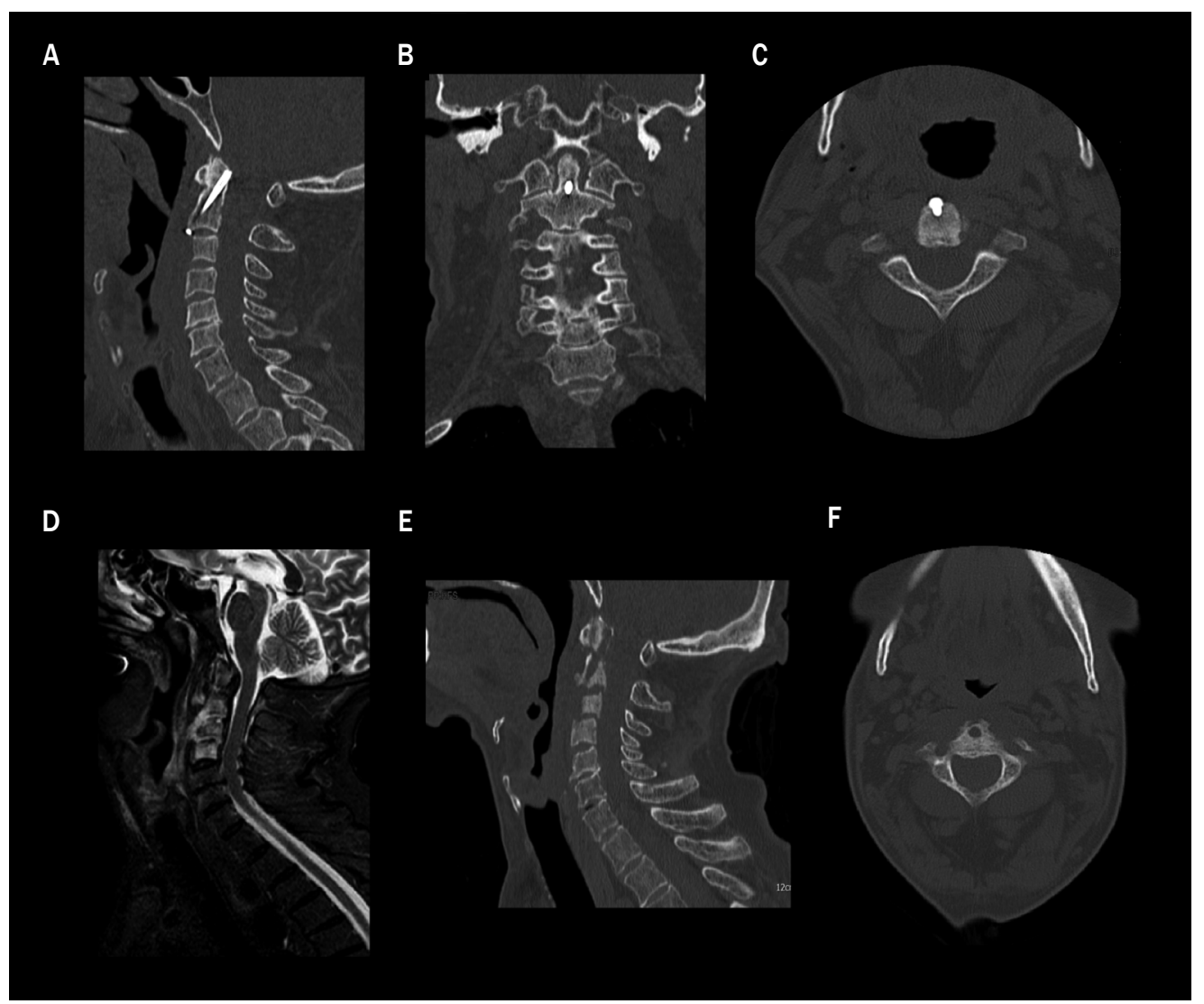

Figure 1. A. Postoperative sagittal CT - proper position of screw fixation; B. Postoperative coronal CT; C. Postoperative transversal CT; D. Postoperative saggital MRI - fistula canal in posterior pharynx extends into the larynx. Diffuse inflammatory of C2-C4 infiltrates into intervertebral discs; E. Postoperative sagittal CT - after screw extrusion. Fracture fissure with signs of osteolysis; F. Postoperative transversal CT

\section{Clinical presentation}

A patient was admitted to the neurosurgery department due to a type II odontoid fracture. This injury was the result of a fall down stairs five days earlier. On admission, the patient complained of neck and arm pain. A neurological examination revealed no abnormalities. The patient underwent ASOF. Control CT presented an acceptable screw position. Ten weeks after primary surgery, the patient returned to hospital for expectorating screw fixations (Fig. 1 A-C). The patient did not report any complaints. In laryngological examination, there were no signs of a fistula. Also gastroscopy was normal, with no signs of a fistula, bleeding, or wounds. While in MRI (Fig. 1 D), features of diffuse inflammation and signs of fistula were described. As a conservative treatment, a stiff cervical collar was used. Parenteral nutrition and antibiotic therapy were introduced. There was no pathology in thorax and cervical CT (Fig. 1 E-F). The patient was admitted to the thoracic surgery department to complete the examination. After three months, the patient was admitted to the neurosurgery department due to a primary non-union fracture. Posterior stabilisation of the $\mathrm{C} 1$ pedicle and $\mathrm{C} 2$-side mass were completed with a $\mathrm{C} 1-\mathrm{C} 2$ spondylodesis. In a postoperative exam, the CT implant position was correct. The patient did not report any complaints. A neurological examination revealed no abnormalities.

\section{Discussion}

A type II odontoid fracture in the Anderson and D'Alonso classification is the most frequent type of fracture in elderly patients, and is estimated at $10-15 \%$ of all cervical fractures $[1,6,9]$. In cases of non-union following conservative treatment, which is estimated to comprise from $20-56 \%$, surgical treatment is widely recommended as the treatment of choice $[1,3]$. The fusion rate in cases of anterior odontoid screw fixation is estimated to be more than $90 \%[1,3,6]$. There is no conclusive evidence that patient age increases the risk of non-union fractures. Tian et al. estimated that the risk of non-union fractures is about $6 \%$ in younger patients and $25 \%$ in patients who are over 50 . This is a result of osteoporosis and diminished bone quality. According to the authors, patients who are aged over 70 have the highest risk of non-union fractures [1]. The treatment method of choice is AOSF. This method provides 
immediate stabilisation. Additionally, it does not limit the mobility of the spine in the $\mathrm{C} 0-\mathrm{C} 2$ segment and eliminates the high risk of vertebral artery damage. This technique results in high fusion rates that range from $89 \%$ to $100 \%$ [6]. The general risk of non-union fractures is estimated at $10 \%$ [1].

Surgical treatment of type II odontoid fractures is mainly performed via an anterolateral approach. The most common complications are associated with this approach. Specific complications related to anatomic areas are postoperative dysphagia (10\%) or hoarseness $(1.2 \%)$, wound haematomas $(0.2 \%)$, and spinal cord injury (up to $0.2 \%$ ). The overall rate of infection is $0.2 \%$. About $5 \%$ of patients need revision surgery [1].

One possible complication is oesophageal perforation. The overall risk is estimated at less than $1 \%[1,2]$. Acute perforation is the result of an intraoperative oesophagus injury which may be the result of sharp-edged surgical instruments, implants or bones, or aggressive surgery exposure. Delayed perforation may be a consequence of local tissue necrosis, erosion or an inflammatory process in this area $[2,8]$. Bones or screws, especially malpositioned or extruded, cause chronic compression or repetitive friction that can lead to local ischaemia and necrosis which result in delayed mucosal perforation of the oesophagus $[5,6,8]$. Oesophagus or pharynx perforation can lead to life-threatening complications such as aspiration pneumonia, mediastinitis, pleuritis, pericarditis, systemic sepsis or airway obstruction $[2,4,5,7]$. The average mortality rate is estimated at $20-50 \%[4,5]$.

Symptoms that may suggest oesophageal perforation are fever, difficulty in swallowing (dysphagia or odynophagia), weight loss, painful neck swelling or subcutaneous emphysema of the neck $[7,8]$. Additionally, patients may experience foreign body sensation or a persistent cough [6]. Notwithstanding this, perforation may be asymptomatic. Only individual cases of oral screw extrusion have been described [8].

Suspected possible factors of screw extrusion are screw malposition and local infection $[5,6,10]$. Statistically, authors have reported that AOSF failure is a result of osteoporosis or poor bone quality, nonoptimal fracture compression or reduction, and non-union fractures $[5,7,10]$. Cho et al. and Koivikko et al. reported a strong influence of treatment delay and fracture gap width on surgery failure $[11,12]$.

In small perforations of less than $1 \mathrm{~cm}$, conservative treatment is preferred [8]. Treatment includes solely extraoral nutrition and antibiotic therapy $[7,8]$. Cases of spontaneous healing and recovery have been reported [5]. Larger defects require surgical revision. Surgical methods of treatment include primary suture, microlaryngological transpharyngeal endoscopic techniques, and vasculised flap implantation connected with implant removal [6-8].

\section{Conclusion}

It is good practice to closely observe patients after cervical spine. Nonspecific symptoms such as fever, foreign body sensation, persistent cough or difficulty in swallowing can be the first symptoms of oesophagus perforation. Patients with a suspected oesophageal perforation should be diagnosed and treated with particular care.

Acknowledgements: None.

Conflict of interest: None.

Funding information and financial support: None.

Disclosures: None.

Patient's consent: Yes.

\section{References}

1. Tian NF, Hu XQ, Wu $\sqcup$, et al. Pooled analysis of non-union, re-operation, infection, and approach related complications after anterior odontoid screw fixation. PLoS One. 2014; 9(7): e103065, doi: 10.1371/ journal.pone.0103065, indexed in Pubmed: 25058011.

2. Kau RL, Kim N, Hinni ML, et al. Repair of esophageal perforation due to anterior cervical spine instrumentation. Laryngoscope. 2010; 120(4): 739-742, doi: 10.1002/lary.20842, indexed in Pubmed: 20232412.

3. Nourbakhsh A, Shi R, Vannemreddy P, et al. Operative versus nonoperative management of acute odontoid Type II fractures: a meta-analysis. J Neurosurg Spine. 2009; 11(6): 651-658, doi: 10.3171/2009.7.SPINE0991, indexed in Pubmed: 19951016.

4. Bohlman HH. Complications of treatment of fracture dislocations of the cervical spine. In: Complications in orthopedic surgery, ed. $\mathrm{CH}$ Epps, JB Lippincott. 1978; 2: 622-623.

5. Lee JS, Kang DH, Hwang SH, et al. Oral extrusion of screw after anterior cervical interbody fusion. J Korean Neurosurg Soc. 2008; 44(4): 259-261, doi: 10.3340/jkns.2008.44.4.259, indexed in Pubmed: 19096688.

6. Lee EJ, Jang JW, Choi SHo, et al. Delayed pharyngeal extrusion of an anterior odontoid screw. Korean J Spine. 2012; 9(3): 289-292, doi: 10.14245/kjs.2012.9.3.289, indexed in Pubmed: 25983835.

7. Chun HJ, Bak KH, Kang TH, et al. Rod migration into the posterior fossa after harms operation : case report and review of literatures. J Korean Neurosurg Soc. 2010; 47(3): 221-223, doi: 10.3340/ jkns.2010.47.3.221, indexed in Pubmed: 20379477.

8. Kim SJ, Ju CII, Kim DM, et al. Delayed esophageal perforation after cervical spine plating. Korean J Spine. 2013; 10(3): 174-176, doi: 10.14245/kjs.2013.10.3.174, indexed in Pubmed: 24757482.

9. Anderson LD, D'Alonzo RT, Anderson LD, et al. Fractures of the odontoid process of the axis. J Bone Joint Surg Am. 1974; 56(8): 1663-1674, indexed in Pubmed: 4434035.

10. Osti M, Philipp H, Meusburger B, et al. Analysis of failure following anterior screw fixation of Type II odontoid fractures in geriatric patients. Eur Spine J. 2011; 20(11): 1915-1920, doi: 10.1007/s00586-0111890-7, indexed in Pubmed: 21728075.

11. Cho DC, Sung JK. Analysis of risk factors associated with fusion failure after anterior odontoid screw fixation. Spine (Phila Pa 1976). 2012; 37(1): 30-34, doi: 10.1097/BRS.0b013e31820e6418, indexed in Pubmed: 21228750.

12. Koivikko MP, Kiuru MJ, Koskinen SK, et al. Factors associated with nonunion in conservatively-treated type-II fractures of the odontoid process. J Bone Joint Surg Br. 2004; 86(8): 1146-1151, doi: 10.1302/0301-620x.86b8.14839, indexed in Pubmed: 15568528. 